\title{
Novel hematological biomarkers predict survival in renal cell carcinoma patients treated with nephrectomy
}

Paweł Rajwa ${ }^{1}$, Marcin Życzkowski ${ }^{1}$, Andrzej Paradysz ${ }^{1}$, Monika Slabon-Turska ${ }^{1}$, Kamil Suliga ${ }^{1}$, Kamil Bujak2, Piotr Bryniarski ${ }^{1}$

\begin{abstract}
${ }^{1}$ Department of Urology, School of Medicine with the Division of Dentistry in Zabrze, Medical University of Silesia in Katowice, Zabrze, Poland

${ }^{2} 3^{\text {rd }}$ Department of Cardiology, School of Medicine with the Division of Dentistry in Zabrze, Medical University of Silesia in Katowice, Silesian Center for Heart Diseases, Zabrze, Poland
\end{abstract}

Submitted: 10 May 2017

Accepted: 16 August 2017

Arch Med Sci 2020; 16 (5): 1062-1071

DOI: https://doi.org/10.5114/aoms.2017.70250

Copyright @ 2017 Termedia \& Banach

\begin{abstract}
Introduction: The association between novel blood-based inflammatory indices and patient survival has been reported with reference to various cancers. The aim of this study was to investigate the prognostic value of preoperative platelet-lymphocyte ratio (PLR), neutrophil-lymphocyte ratio (NLR), derived neutrophil-lymphocyte ratio (dNLR) and lymphocyte-monocyte ratio $(L M R)$ in patients with renal cell carcinoma (RCC) treated with nephrectomy. Material and methods: From 2003 to 2012, 455 patients who underwent partial or radical nephrectomy for RCC were enrolled in the study. The study endpoints were overall survival (OS) and cancer-specific survival (CSS).

Results: The median follow-up was 70 months. Groups of patients with high levels of PLR, NLR and dNLR and a low level of LMR more often underwent radical nephrectomy, had a higher cancer stage in the TNM classification, and were more frequently diagnosed with tumor necrosis in histopathological examination. Both cancer-specific mortality and overall mortality were significantly higher in patients with high PLR, NLR and dNLR and low LMR. Multivariate analysis of CSS, adjusted for standard clinicopathological factors, identified only $\operatorname{dNLR}(p=0.006)$ as an independent prognostic factor. $\operatorname{PLR}(p=0.0002), \operatorname{dNLR}(p=0.0003)$ and NLR $(p=0.002)$, but not LMR $(p=0.1)$, achieved prognostic significance in multivariable analysis regarding OS.

Conclusions: Only dNLR was an independent prognostic factor for CSS and OS. Nevertheless, our study indicates that all examined complete blood count-based biomarkers may be useful tools in managing RCC patients treated with a surgical approach.
\end{abstract}

Key words: renal cell cancer, renal cell carcinoma, platelet-lymphocyte ratio, neutrophil-lymphocyte ratio, derived neutrophil-lymphocyte ratio, lymphocyte-monocyte ratio.

\section{Introduction}

It is estimated that kidney cancer will be the $6^{\text {th }}$ most frequently diagnosed malignancy amongst men and the $10^{\text {th }}$ amongst women in 2017 in the United States [1]. Renal cell carcinoma (RCC), as the most common kidney cancer, comprises approximately $90 \%$ of all renal neoplasms [2]. In recent years the world incidence of RCC has increased, which might be

\author{
Corresponding author: \\ Pawet Rajwa \\ Department of Urology \\ School of Medicine \\ with the Division \\ of Dentistry \\ Medical University of Silesia \\ 13-15 3 Maja St \\ 41-800 Zabrze, Poland \\ Phone: +48323704454 \\ E-mail: pawelgrajwa@gmail. \\ com
}


associated with rising prevalence of well-known and verified risk factors such as tobacco smoking, obesity and hypertension [3, 4]. Despite the unquestionable progress that has been made in diagnosis and treatment of RCC, the 5-year survival still remains unsatisfactory [5]. The surgical approach regarding partial (PN) and radical (RN) nephrectomy should be a first-choice treatment in RCC patients [5].

Nowadays, rapidly growing evidence confirms that inflammation plays a vital role at every stage of tumor development: from initiation to metastasis [6]. Renal cell cancer, being one of the well-documented inflammation-related neoplasms, modulates the host immune system for its growth and metastasis [7, 8]. Considering the number and the heterogeneity of RCC prognostic factors and the interactions between renal cell carcinoma and immune cells, searching for widely available and reliable inflammatory biomarkers seems essential [7, 9]. Up to now, numerous studies have reported that some non-specific indicators derived from complete blood count (CBC) give added prognostic value in the assessment of various diseases, including renal cell carcinoma [10-16]. Amongst the most promising indices that are based on absolute values of circulating inflammatory cells, platelet-lymphocyte ratio (PLR), neutrophil-lymphocyte ratio (NLR), derived neutrophil-lymphocyte ratio (dNLR) and lymphocyte-monocyte ratio (LMR) are being particularly deeply investigated. To date, only a few studies assessing the prognostic value of PLR, NLR, dNLR and LMR in patients diagnosed with RCC and treated with radical ( $R N)$ or partial (PN) nephrectomy have been published [17-20].

Therefore, the aim of our study was to establish the prognostic value of PLR, NLR, dNLR and LMR in a relatively large cohort of unselected surgically treated RCC subjects.

\section{Material and methods}

We retrospectively investigated data from 571 patients treated with radical or partial nephrectomy between 2003 and 2012 in a single tertiary academic centre in Poland. Only patients with histopathologically confirmed renal cell carcinoma were enrolled in this research. Study subjects with non-RCC kidney neoplasm $(n=69)$, other solid tumors $(n=7)$, hematological diseases $(n=8)$, severe inflammatory conditions $(n=31)$ and those who died due to perioperative complications $(n=1)$ were excluded. The final cohort consisted of 455 patients. Data regarding sex, age, date of surgery, comorbidities, operative method (partial vs. radical) and cancer characteristics were obtained from medical records. PLR, NLR, dNLR and LMR were obtained from preoperative CBCS, which were performed at a median of 3 (min.: 1; max.: 13) days prior to nephrectomy. Following the study of Proctor et al. dNLR was calculated as neutrophil count to (white cell count minus neutrophil count) [21].

Due to the change in tumor staging system during the observational period, TNM classification was assessed in compliance with the American Joint Committee on Cancer, $7^{\text {th }}$ edition (2010) [22]. Tumor grading was performed according to the Fuhrman system [23]. The primary study endpoint was cancer-specific survival (CSS), defined as time (months) from surgical intervention to cancer-related death or the date of the end of follow-up, which was May 15, 2016. The secondary study end-point was overall survival (OS), regarded as time from surgery to death or the date of the end of follow-up. We obtained mortality data from the Centre for Document Personalization of the Polish Ministry of Interior and Administration, which stores, among other data, the exact date of death of every Polish citizen since the mid1980s. Cause of death was obtained from the Polish National Cancer Registry and the National Health Fund by meticulous analysis of received patients' data, which included the last hospitalization records, main diagnosis of the underlying disease, concomitant illnesses, and direct and indirect cause of death. Furthermore, we made an attempt to contact the relatives of dead patients with a $61 \%$ success rate and complete congruence with data received from the aforementioned institutions. Overall, complete follow-up data were available for 443 (97.36\%) patients finally included in this study. The local Ethics Committee approved the study in 2016 under the number KNW/0022/KB/91/16.

\section{Statistical analysis}

The normality of continuous variable distribution was tested with the Shapiro-Wilk test. Continuous variables were not distributed normally and are presented as median (interquartile range). Dichotomous variables are presented as percentages. Optimal cut-off values of PLR, NLR, dNLR and LMR for predicting CSS and OS were determined using receiver operating characteristic (ROC) curve analysis. Due to the fact that there are no generally established normal ranges of the inflammatory indices' values, we divided the patients into groups with low and high PLR, NLR, dNLR and LMR according to optimal cut-off points. High and low PLR, NLR and dNLR were regarded as higher than or equal to and lower than optimal thresholds, respectively. Low and high LMR was defined as lower than or equal to and higher than optimal cutoffs, respectively. To test the differences between groups, the Mann-Whitney $U$ or the Kruskal-Wallis and post hoc Dunn tests were used for continu- 
Table I. Patients' baseline characteristics, pathological findings and long-term outcomes

\begin{tabular}{|c|c|}
\hline Parameter & Value \\
\hline Sex (male/female) & $53.4 \% / 46.6 \%$ \\
\hline Age [years] & $62.0(54.0-70.0)$ \\
\hline BMI $\left[\mathrm{kg} / \mathrm{m}^{2}\right]$ & $27.2(24.6-30.4)$ \\
\hline Nephrectomy (partial/radical) & $31.9 \% / 68.1 \%$ \\
\hline \multicolumn{2}{|l|}{ Histology: } \\
\hline Clear cell & $84.2 \%$ \\
\hline Chromophobe & $8.7 \%$ \\
\hline Papillary & $5.0 \%$ \\
\hline Cystic & $1.8 \%$ \\
\hline Undifferentiated & $0.2 \%$ \\
\hline $\begin{array}{l}\text { Sarcomatoid feature } \\
\text { (present/absent) }\end{array}$ & $3.4 \% / 96.6 \%$ \\
\hline \multicolumn{2}{|l|}{ pT stage: } \\
\hline pT1 & $63.0 \%$ \\
\hline рT2 & $12.0 \%$ \\
\hline рT3 & $24.0 \%$ \\
\hline pT4 & $1.0 \%$ \\
\hline \multicolumn{2}{|l|}{ N stage: } \\
\hline NO & $93.6 \%$ \\
\hline N1 & $6.4 \%$ \\
\hline \multicolumn{2}{|l|}{ M stage: } \\
\hline MO & $93.8 \%$ \\
\hline M1 & $6.2 \%$ \\
\hline Tumor necrosis (present/absent) & $17.1 \% / 82.9 \%$ \\
\hline Tumor size $[\mathrm{mm}]$ & $50.0(35.0-70.0)$ \\
\hline Hemoglobin [g/dl] & $13.8(12.7-14.8)$ \\
\hline PLR & $126.5(96.4-170.8)$ \\
\hline NLR & $2.1(1.6-2.9)$ \\
\hline$d N L R$ & $1.5(1.1-2.0)$ \\
\hline LMR & $4.3(3.1-5.7)$ \\
\hline Overall mortality & $40 \%$ \\
\hline Cancer-specific mortality & $21 \%$ \\
\hline
\end{tabular}

Continuous variables are presented as median (interquartile range) Dichotomous variables are presented as percentages. BMI - body mass index, PLR - platelet-lymphocyte ratio, NLR - neutrophillymphocyte ratio, $d N L R$ - derived neutrophil-lymphocyte ratio, $L M R$ - lymphocyte-monocyte ratio.

ous variables. Categorical variables were compared using the $\chi^{2}$ test. The associations between groups and CSS and OS were analyzed using the Kaplan-Meier method with log-rank testing. The
Cox proportional hazards regression model was applied to perform univariate and multivariate analysis. PLR, NLR, dNLR and LMR were included in the Cox proportional hazard regression analysis as continuous variables. Variables that reached a $p$-value lower than 0.05 in the univariate analysis were entered into the multivariable analysis. A Bonferroni corrected $p$-value $<0.0125(0.05 / 4)$ was considered as the threshold of statistical significance. Statistical analyses were performed using Statistica 12 software with the Medical Bundle (StatSoft Inc., Tulsa, Oklahoma, USA).

\section{Results}

Baseline demographic and clinical patients characteristics are summarized in Table I. Median follow-up was 70 months. During the follow-up period 179 (40\%) patients died from any cause and among them 91 patients ( $21 \%$ of all patients) died from RCC-related causes.

The distribution of PLR, NLR, dNLR and LMR values in the whole group of patients is presented in Figure 1. Optimal cut-off values of PLR, NLR, dNLR and LMR in predicting CSS were 176.32, 2.45, 1.43, and 3.25 , respectively. Regarding OS, optimal cutoff values were 168.46 for PLR, 2.14 for NLR, 1.52 for $\mathrm{dNLR}$, and 3.75 for LMR. The ROC curves for the aforementioned biomarkers in prediction of CSS and OS are depicted in Figures 2 and 3, respectively.

Patients with high values of PLR and low values of LMR were older. There were no significant differences regarding age among patients with high and low values of NLR and dNLR. In addition, groups of patients with high levels of PLR, NLR, $d N L R$ and low level of LMR more often underwent radical nephrectomy, had higher cancer stage in TNM classification and were more frequently diagnosed with tumor necrosis in histopathological examination (Table II). Both cancer-specific mortality and overall mortality were significantly higher in patients with high PLR, NLR, dNLR and low LMR (Table II, Figures 4 and 5). Study subjects who died from cancer had significantly higher values of PLR, NLR and dNLR and lower values of LMR, when compared to patients who survived or died from cancer-unrelated causes (Table III).

In the univariate Cox regression model, all analyzed measurements were significantly associated with CSS and OS (Tables IV and V). After adjusting for clinical and histopathological covariates, only dNLR met the Bonferroni-corrected threshold of significance in predicting both CSS and OS. PLR and NLR achieved independent predictor status only for OS (Tables IV and V).

\section{Discussion}

A growing body of evidence supports the theory that new pre-treatment blood-based indica- 
A

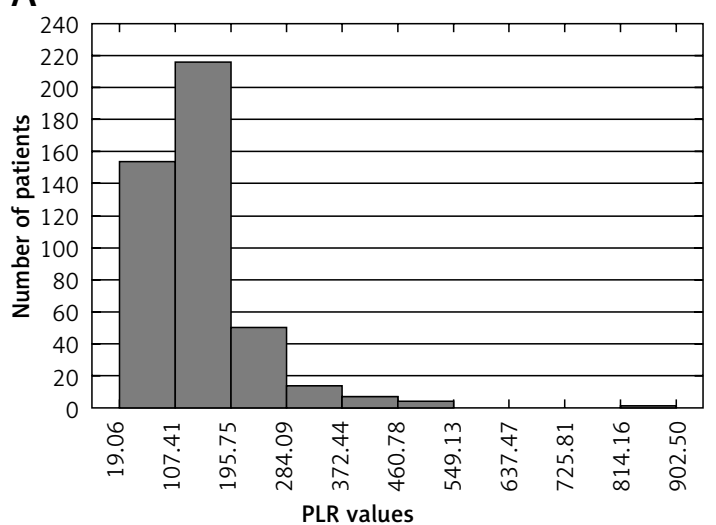

C

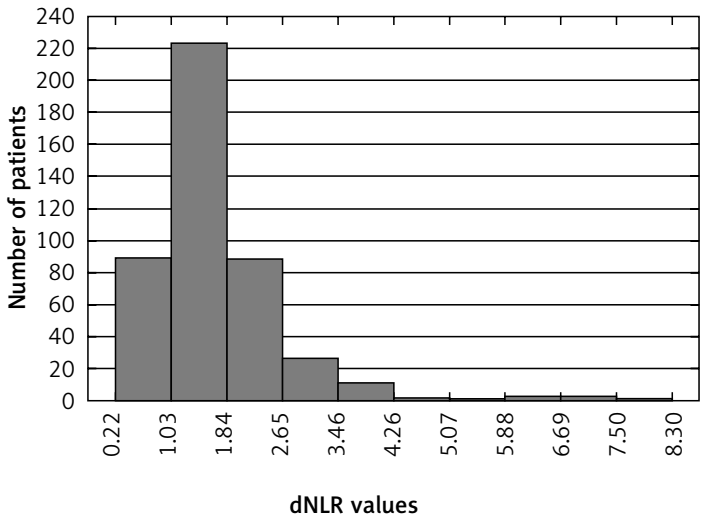

B

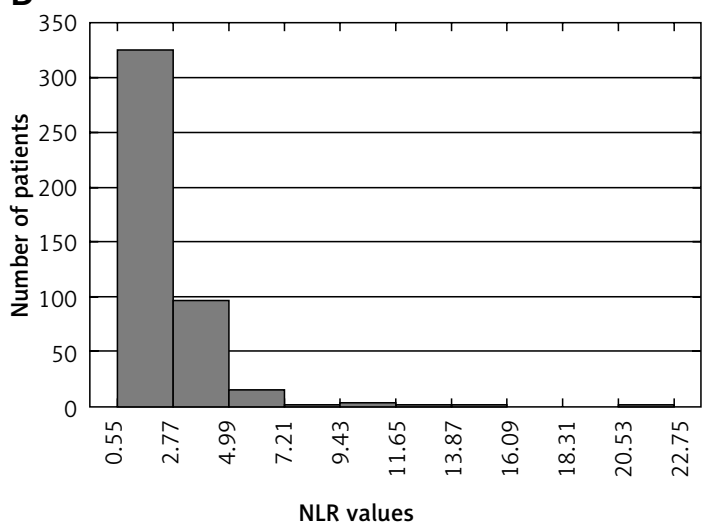

D

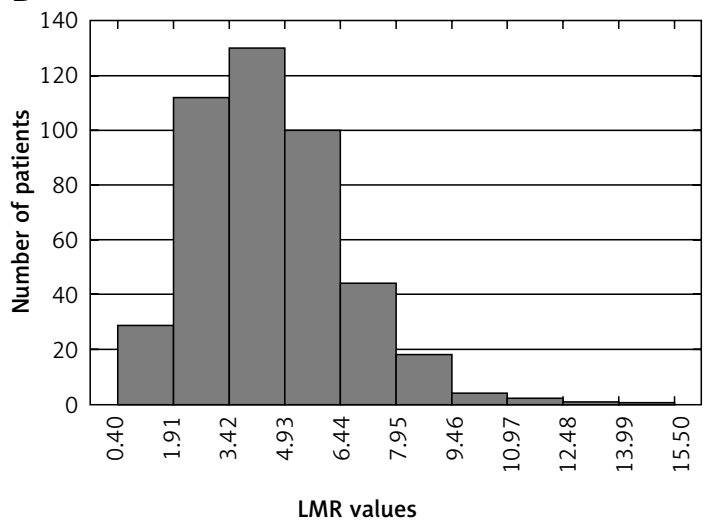

Figure 1. Distribution of PLR (A), NLR (B), dNLR (C) and LMR (D) values in the whole group of patients

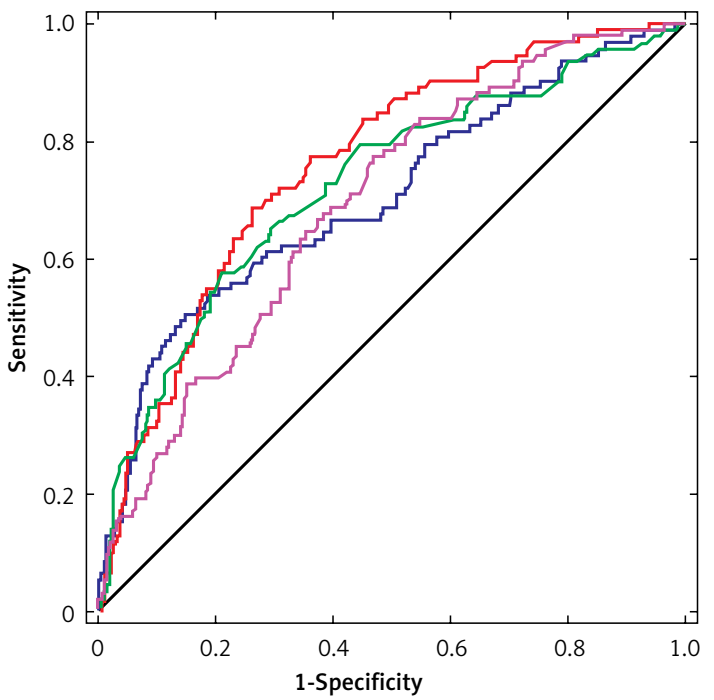

- PLR (AUC: $0.704 ; 95 \% \mathrm{Cl}: 0.64-0.767$ )

NLR (AUC: $0.759 ; 95 \% \mathrm{Cl}: 0.706-0.812)$

- dNLR (AUC: $0.691 ; 95 \% \mathrm{Cl}: 0.634-0.749)$

— LMR (AUC: $0.724 ; 95 \% \mathrm{Cl}: 0.663-0.786)$

- Reference line

Figure 2. Receiver operating characteristic (ROC) curves for PLR, NLR, $d N L R$ and $L M R$ in predicting CCS

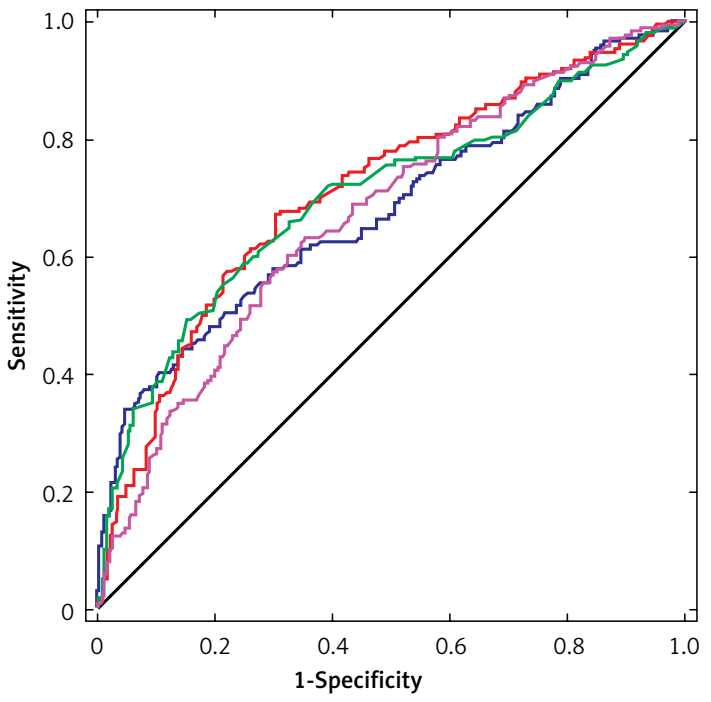

PLR (AUC: 0.68; 95\% Cl: 0.627-0.732)

NLR (AUC: $0.713 ; 95 \% \mathrm{Cl}: 0.663-0.763$ )

dNLR (AUC: $0.673 ; 95 \% \mathrm{Cl}: 0.621-0.724$ )

— LMR (AUC: $0.703 ; 95 \%$ Cl: $0.65-0.755$ )

- Reference line

Figure 3. Receiver operating characteristic (ROC) curves for PLR, NLR, dNLR and LMR in predicting OS 


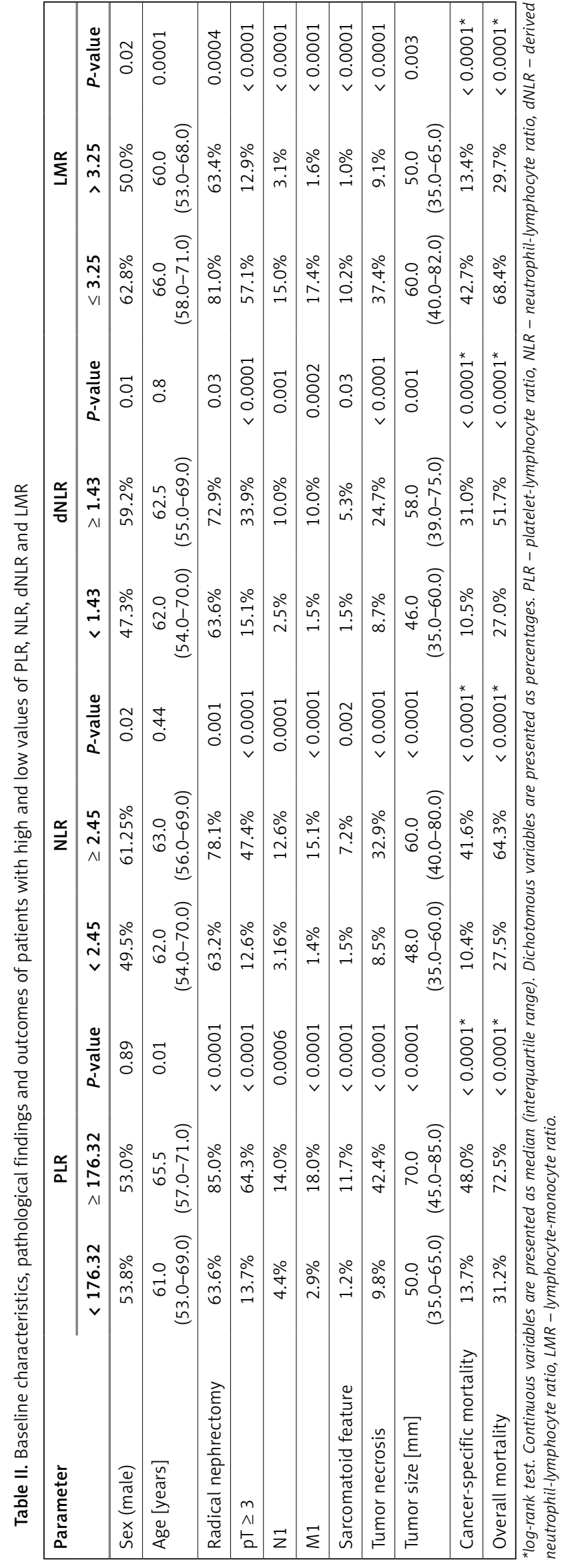

tors may be additional tools in understanding and managing renal cell carcinoma, which is a particularly unpredictable tumor $[9,11]$. Thus far, data regarding the prognostic value of PLR, NLR, dNLR, and LMR in RCC patients treated with nephrectomy are sparse and inconclusive. Most of the previously conducted studies focused on the prognostic value of one or two indicators. Apart from one study, conducted by Gu et al., who analyzed the association between all four inflammatory bloodbased measurements and OS in 103 sarcomatoid RCC patients, no study to date has evaluated them simultaneously in one cohort of renal cell carcinoma patients [24]. Moreover, it must be pointed out that the vast majority of studies used PLR, NLR, dNLR and LMR coded as categorical variables in the Cox regression model, which is associated with multiple limitations, including a higher risk of type I error [25, 26].

Regarding the obtained results, there are several pivotal findings of our study. First, dNLR, which is derived from the absolute number of lymphocytes, monocytes, neutrophils, basophils and eosinophils, is the only measurement that was found to be an independent prognostic factor in our multivariate analyses, irrespective of the chosen end-point. Second, high PLR and NLR levels have been significantly and independently associated with increased risk of overall mortality, but not cancer-specific mortality. This may indicate that PLR and NLR are biomarkers of a generally impaired immune response or comorbidity burden, which may predispose to higher risk of all-cause death, rather than factors specific to a neoplastic process. Third, PLR, NLR, dNLR and LMR have been associated with TNM staging components, which implies that these indicators may not only be useful in evaluating the outcome of patients undergoing nephrectomy, but can also be helpful in the preoperative evaluation of cancer stage.

\section{Pathophysiology}

Since Rudolf Virchow, who in 1863 first suggested that there is a link between inflammation and malignancy, numerous studies have confirmed his hypothesis $[6,27,28]$. Currently, the role of specific types of immune cells in tumor initiation, growth and metastasis is being deeply investigated [29-32]. Lymphocytes, whose absolute count is a common component of CBC-derived biomarkers, are the basic line of defense against tumor cells. Cancer-induced lymphocytopenia may be a result of excessive expression of proapoptotic ligands, such as the Fas ligand, transforming growth factor $\beta$ or programmed death-ligand 1 produced by cancer cells $[28,29,33]$. Also, primary reduced lymphocyte number might be in itself responsible for the impaired immune response to developing 
A

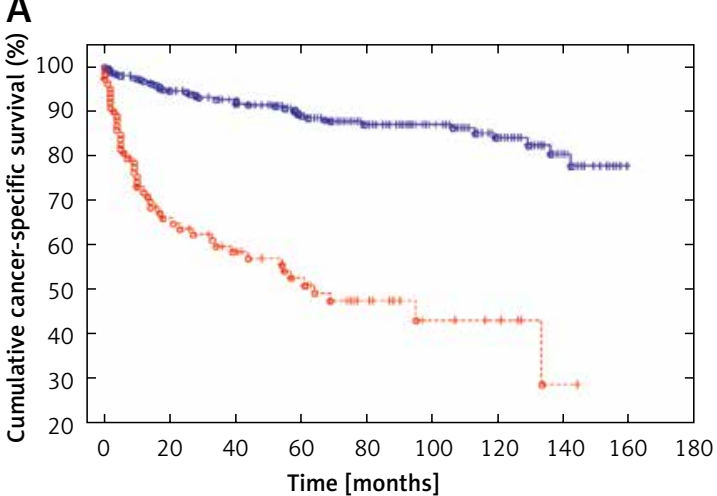

- Low PLR ------ High PLR

C

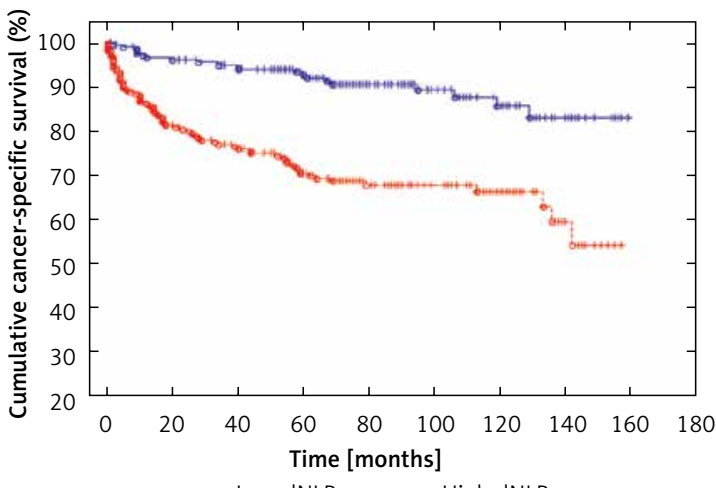

— Low dNLR ----- High dNLR

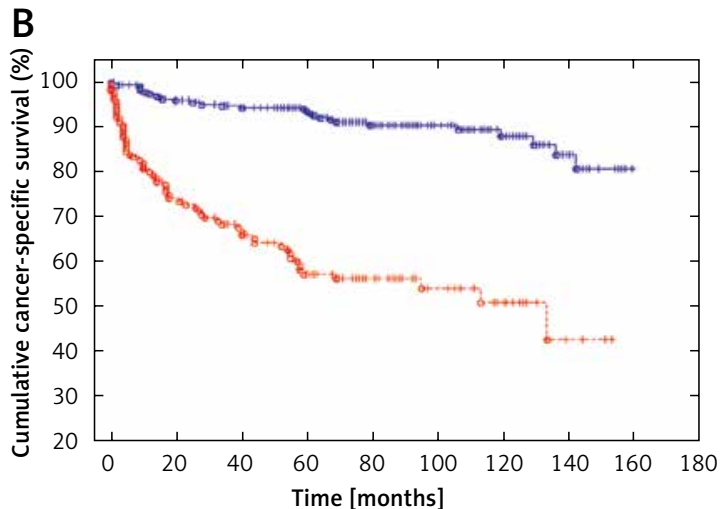

— Low NLR ----- High NLR

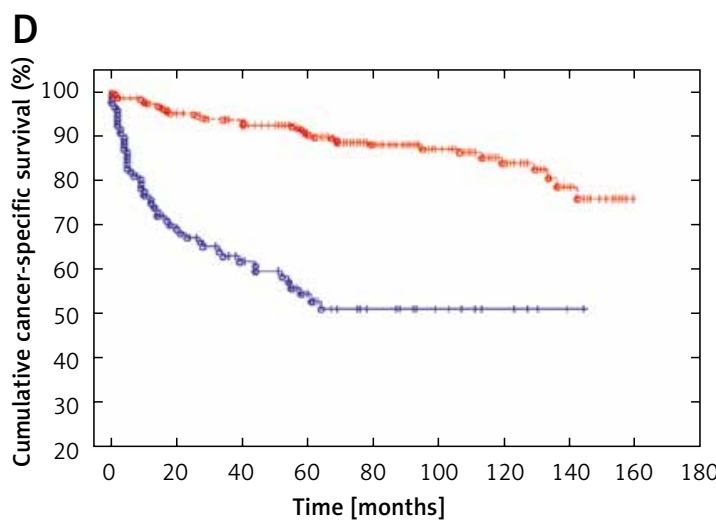

— Low LMR ----- High LMR

Figure 4. Cancer-specific survival in patients with low and high values of PLR (A), NLR (B), dNLR (C) and LMR (D) Kaplan-Meier curves

A

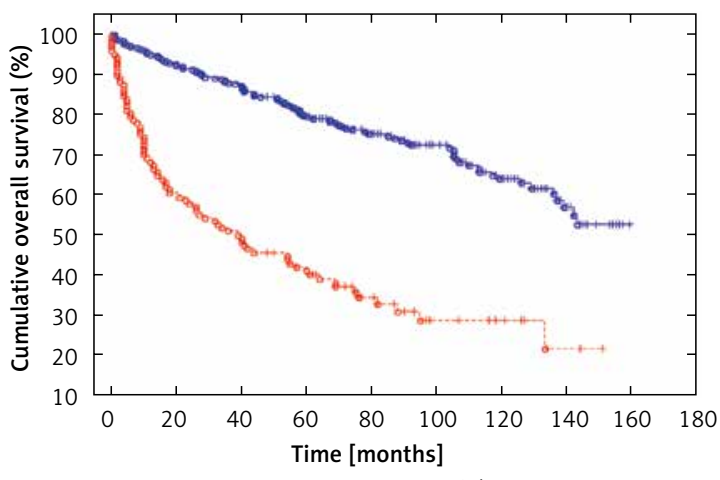

C

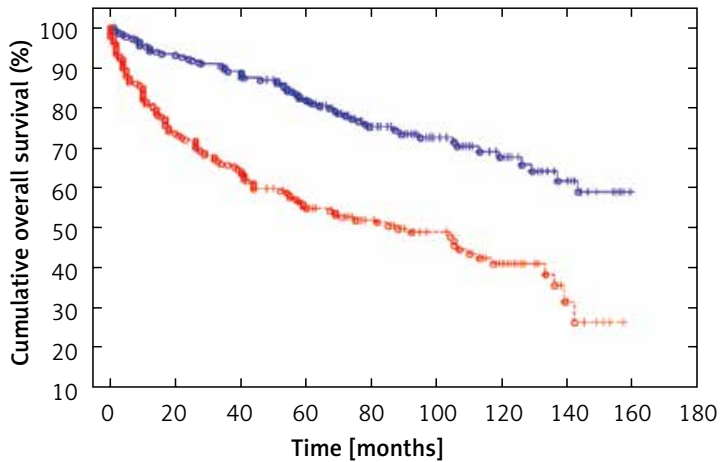

B

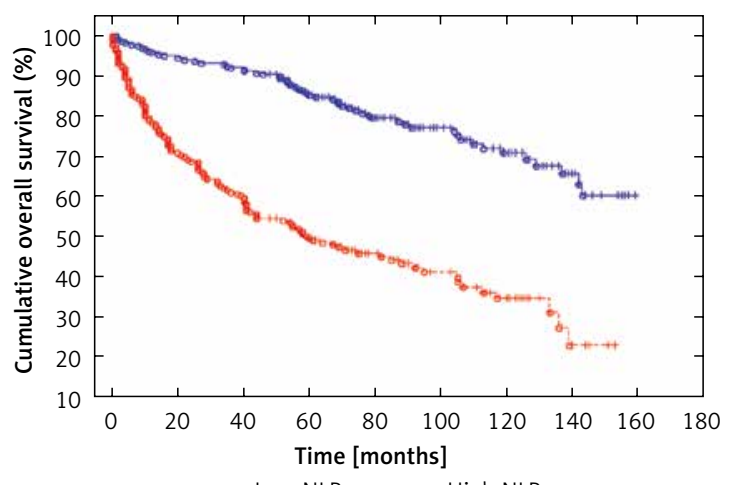

- Low NLR ----- High NLR

D

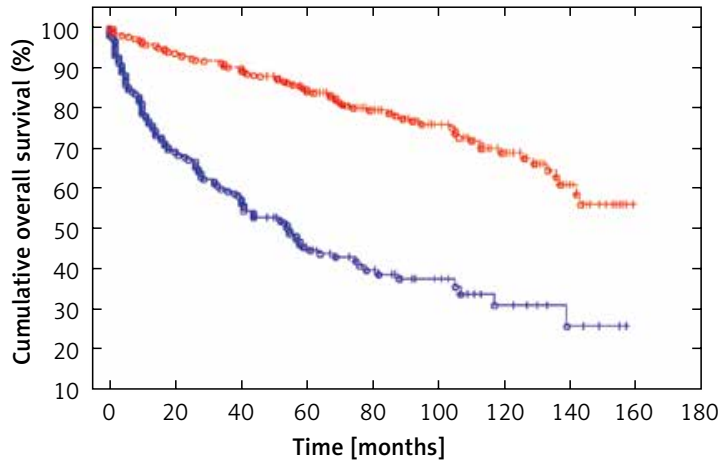

- Low LMR ----- High LMR

Figure 5. Overall survival in patients with low and high values of PLR (A), NLR (B), dNLR (C) and LMR (D) Kaplan-Meier curves 
Table III. Comparison of PLR, NLR, dNLR and LMR values between patients who survived and died during follow-up

\begin{tabular}{|lccccccc|}
\hline Parameter & $\begin{array}{c}\text { Survivors } \\
\boldsymbol{N}=264\end{array}$ & $\begin{array}{c}\text { Cancer-spe- } \\
\text { cific deaths } \\
\boldsymbol{N}=91\end{array}$ & $\begin{array}{c}\text { Cancer- } \\
\text { unrelated } \\
\text { deaths } \\
N=88\end{array}$ & $\begin{array}{c}P \text {-value } \\
\text { (all groups) }\end{array}$ & $\begin{array}{c}P \text {-value } \\
\text { (survivors } \\
\text { vs. cancer- } \\
\text { specific } \\
\text { deaths) }\end{array}$ & $\begin{array}{c}P \text {-value } \\
\text { (survivors } \\
\text { vs. cancer- } \\
\text { unrelated } \\
\text { deaths) }\end{array}$ & $\begin{array}{c}P \text {-value } \\
\text { (cancer-spe- } \\
\text { cific deaths } \\
\text { vs. cancer- } \\
\text { unrelated } \\
\text { deaths) }\end{array}$ \\
\hline PLR & $\begin{array}{c}117.8 \\
(90.9-147.1)\end{array}$ & $\begin{array}{c}176.3 \\
(117.0-233.1)\end{array}$ & $\begin{array}{c}138.3 \\
(96.1-186.9)\end{array}$ & $<0.0001$ & $<0.0001$ & 0.003 & 0.02 \\
\hline NLR & $\begin{array}{c}1.82 \\
(1.39-2.36)\end{array}$ & $\begin{array}{c}2.92 \\
(2.18-4.09)\end{array}$ & $\begin{array}{c}2.23 \\
(1.6-3.31)\end{array}$ & $<0.0001$ & $<0.0001$ & 0.002 & 0.0004 \\
\hline dNLR & 1.33 & 1.77 & 1.61 & $<0.0001$ & $<0.0001$ & 0.003 & 0.07 \\
\hline LMR & $(1.0-1.7)$ & $(1.45-2.45)$ & $(1.21-2.24)$ & & & & \\
& 4.8 & 3.07 & 3.76 & $<0.0001$ & $<0.0001$ & 0.0004 & 0.02 \\
\hline
\end{tabular}

Continuous variables are presented as median (interquartile range). PLR - platelet-lymphocyte ratio, NLR - neutrophil-lymphocyte ratio, $d N L R$ - derived neutrophil-lymphocyte ratio, LMR - lymphocyte-monocyte ratio.

Table IV. Relationship between PLR, NLR, dNLR, LMR and CSS in patients with RCC who underwent nephrectomy univariate and multivariate analysis

\begin{tabular}{|lcccccc|}
\hline Parameter & \multicolumn{3}{c}{ Univariate analysis } & \multicolumn{3}{c|}{ Multivariate analysis* $^{*}$} \\
\cline { 2 - 8 } & HR & $95 \% \mathrm{Cl}$ & $P$-value & HR & $95 \%$ Cl & $P$-value \\
\hline PLR & 1.005 & $1.004-1.006$ & $<0.0001$ & 1.002 & $1.0-1.003$ & 0.094 \\
\hline NLR & 1.183 & $1.127-1.241$ & $<0.0001$ & 1.041 & $0.965-1.124$ & 0.299 \\
\hline dNLR & 1.628 & $1.417-1.869$ & $<0.0001$ & 1.322 & $1.083-1.614$ & 0.006 \\
\hline LMR & 0.595 & $0.515-0.688$ & $<0.0001$ & 0.937 & $0.807-1.089$ & 0.399 \\
\hline
\end{tabular}

${ }^{\star}$ Adjusted for: age (continuous), higher Fuhrman grade (G3-4 vs. G1-2), higher pT category (pT3-4 vs. pT1-2), lymph node involvement (presence vs. absence), distant metastasis (presence vs. absence) and tumor necrosis (presence vs. absence). PLR - platelet-lymphocyte ratio, NLR - neutrophil-lymphocyte ratio, $d N L R$ - derived neutrophil-lymphocyte ratio, LMR - lymphocyte-monocyte ratio, CSS - cancerspecific survival, HR - hazard ratio, Cl - confidence interval.

Table V. Relationship between PLR, NLR, dNLR, LMR and OS in patients RCC who underwent nephrectomy - univariate and multivariate analysis

\begin{tabular}{|c|c|c|c|c|c|c|}
\hline \multirow[t]{2}{*}{ Parameter } & \multicolumn{3}{|c|}{ Univariate analysis } & \multicolumn{3}{|c|}{ Multivariate analysis* } \\
\hline & HR & $95 \% \mathrm{Cl}$ & $P$-value & HR & $95 \% \mathrm{Cl}$ & $P$-value \\
\hline PLR & 1.005 & $1.004-1.006$ & $<0.0001$ & 1.003 & $1.001-1.004$ & 0.0002 \\
\hline NLR & 1.185 & $1.14-1.232$ & $<0.0001$ & 1.087 & $1.031-1.147$ & 0.002 \\
\hline $\mathrm{dNLR}$ & 1.531 & $1.364-1.718$ & $<0.0001$ & 1.337 & $1.144-1.562$ & 0.0003 \\
\hline LMR & 0.676 & $0.610-0.748$ & $<0.0001$ & 0.915 & $0.823-1.017$ & 0.1 \\
\hline
\end{tabular}

${ }^{\star}$ Adjusted for: age (continuous), higher Fuhrman grade (G3-4 vs. G1-2), higher pT category (pT3-4 vs. pT1-2), lymph node involvement (presence vs. absence), distant metastasis (presence vs. absence) and tumor necrosis (presence vs. absence). PLR - platelet-lymphocyte ratio, NLR - neutrophil-lymphocyte ratio, $d N L R$ - derived neutrophil-lymphocyte ratio, LMR - lymphocyte-monocyte ratio, OS - overall survival, $H R$ - hazard ratio, $\mathrm{Cl}$ - confidence interval.

neoplasms [29]. It has been proven that thrombocytes, whose number is a component of PLR, may be elevated during neoplastic diseases [30]. An increase in absolute platelet number might be linked to tumor cell interleukin-1 or interleukin- 6 secretion, which stimulates megakaryocyte proliferation in bone marrow [30]. Thrombocytes' pro-metastatic role is well established as they secrete numerous factors that facilitate the penetration of cancer cells through blood vessels and induce angiogenesis in metastatic foci [30]. In addition, in kidney cancer patients platelet activation markers, such as P-selectin, are increased, which reflects an enhanced tumor cell-platelet interaction [34]. Circulating monocytes play a pivotal role in host-cancer interplay, as they can differentiate into tumor-associated macrophages (TAMs) [31]. The TAMs, which reside in the tumor microenvironment, secrete vascular endothelial growth factor, epidermal growth factor, fibroblast 
growth factor, platelet-derived growth factor, and transforming growth factor $\beta$, which stimulate angiogenesis, migration, and infiltration, as well as the phenotypic polarization of different immune cells [31, 35-37]. On top of that, according to Komohara et al., increased concentration of TAMs is associated with worse prognosis in kidney cancer patients [38]. Previously, the role of neutrophils was diminished, mainly due to their short lifespan in peripheral blood [32]. Recent studies indicate that neutrophils may live up to $17 \mathrm{~h}$ in the tumor microenvironment, stimulating tumor growth [32]. Furthermore, it is believed that cancer cells - through the interleukin-17-granulocyte colony-stimulating factor axis - induce the release of immature neutrophils, which might have protumorigenic functions [32].

\section{Platelet-lymphocyte ratio}

To our knowledge, only a few studies have appraised the value of PLR in RCC patients undergoing kidney resection $[11,24,39]$. Furthermore, the data are contradictory. Lucca et al. considered the association between PLR and disease-free survival (DFS) of clear cell RCC patients treated with nephrectomy [39]. Categorized, but not continuous PLR was an independent prognostic factor of DFS in multivariable analysis. As mentioned, our analyses have shown that continuous PLR is an independent predictor of OS, but not CSS. However, in a study conducted by Chen et al., categorized PLR did not reach the level of statistical significance for either OS or CSS in multivariable analysis [40].

\section{Neutrophil-lymphocyte ratio}

NLR is one of the best known and first described CBC-derived measurements [12]. The prognostic value of NLR in predicting OS has been confirmed in numerous studies concerning renal cell carcinoma. In a large-scale study of 678 nonmetastatic European RCC patients, NLR was independently associated with OS, but not CSS, which is similar to our results [41]. On the other hand, a study carried out by Viers et al. indicated contradictory results by revealing an increased risk of cancer-specific mortality in patients with a higher pretreatment NLR level [17].

\section{Derived neutrophil-lymphocyte ratio}

In 2012, Proctor et al. introduced a new inflammatory biomarker - dNLR [21]. Due to greater availability and a similar prognostic value in cancer patients, it has been proposed as an alternative biomarker to the well-known NLR [21]. Our results confirm that - out of all analyzed bloodbased measurements - only dNLR reached prog- nostic significance for RCC-specific survival. This is in line with the results of a study by Dalpiaz et al., who first established the prognostic value of dNLR $\geq 2$ in predicting CSS in patients with non-metastatic clear cell RCC [42].

\section{Lymphocyte-monocyte ratio}

Compared with other inflammatory measurements, LMR has been shown to be inversely associated with cancer patients' outcomes [43]. Hutterer et al. examined the influence of low LMR on CSS, OS and metastasis-free survival (MFS) in clear cell RCC patients [43]. Although they reported the association between lower LMR values and CSS, they did not find a similar relationship between OS and MFS. In our study LMR failed to achieve an independent predictor status for CSS and OS in the multivariable model.

\section{Study limitations}

Like every study, our research has a few limitations. To begin with, it is a single-center, retrospective study encompassing only Caucasian patients. In spite of the fact that we excluded patients with recorded severe inflammatory comorbidities, we cannot be certain that there were no subjects with undiagnosed conditions. Furthermore, patients were operated on by multiple surgeons throughout a long period of time. Also, we were not able to obtain data concerning postoperative therapy, which may affect patients' outcomes. Despite these limitations, we present the first study that simultaneously assesses the prognostic value of PLR, NLR, dNLR and LMR in RCC patients treated with nephrectomy. We suggest that further, multi-center and prospective studies are required to confirm our findings.

In conclusion, we believe that our study is the first to simultaneously evaluate preoperative PLR, $\mathrm{dNLR}, \mathrm{NLR}$ and LMR in prognosis of CSS and OS in RCC patients who were treated with nephrectomy. As far as we are concerned, the presented results deserve particular consideration and special attention, because widely investigated PLR, NLR and $L M R$ did not achieve independent predictor status for CSS in RCC patients. Interestingly, dNLR, the value of which had been barely examined, was the only independent variable in the multivariate analysis of OS and CSS. This is also the first study that confirms the prognostic value of PLR regarding OS in a large cohort of RCC study subjects treated with a surgical approach. Our data reveal that noninvasive and simple inflammatory biomarkers may be an additional tool in managing RCC patients. Routinely performed pretreatment $C B C$ testing, in particular platelet, neutrophil, monocyte, lymphocyte and white blood cell counts, when combined with recently implemented ratios, may have significant 
clinical relevance in preoperative mortality risk stratification of RCC patients undergoing partial or radical nephrectomy.

\section{Conflict of interest}

The authors declare no conflict of interest.

\section{References}

1. Siegel RL, Miller KD, Jemal A. Cancer statistics, 2017. CA Cancer J Clin 2017; 67: 7-30.

2. Ljungberg B, Campbell SC, Cho HY, et al. The epidemiology of renal cell carcinoma. Eur Urol 2017; 60: 615-21.

3. King SC, Pollack LA, Li J, et al. Continued increase in incidence of renal cell carcinoma, especially in young patients and high grade disease: United States 2001 to 2010. J Urol 2014; 191: 1665-70.

4. Chow WH, Gridley G, Fraumeni JFJ, Jarvholm B. Obesity, hypertension, and the risk of kidney cancer in men. N Engl J Med 2000; 343: 1305-11.

5. Ljungberg B, Bensalah K, Canfield S, et al. EAU guidelines on renal cell carcinoma: 2014 update. Eur Urol 2015; 67: 913-24.

6. Mantovani A, Allavena P, Sica A, Balkwill F. Cancer-related inflammation. Nature 2008; 454: 436-44.

7. de Vivar Chevez AR, Finke J, Bukowski R. The role of inflammation in kidney cancer. In: Inflammation and Cancer. Advances in Experimental Medicine and Biology. Aggarwal B, Sung B, Gupta S (eds). Springer, Basel 2014

8. Fitzgerald JP, Nayak B, Shanmugasundaram K, et al. Nox4 mediates renal cell carcinoma cell invasion through hypoxia-induced interleukin-6 and -8 production. PLoS One 2012; 7: e30712.

9. Sun M, Shariat SF, Cheng C, et al. Prognostic factors and predictive models in renal cell carcinoma: a contemporary review. Eur Urol 2011; 60: 644-61.

10. Wasilewski J, Pyka L, Hawranek M, et al. Prognostic value of neutrophil to lymphocyte ratio in predicting long-term mortality in patients with ischemic and nonischemic heart failure. Pol Arch Med Wewn 2016; 126: 166-73.

11. Grimes N, Tyson M, Hannan C, Mulholland C. A systematic review of the prognostic role of hematologic scoring systems in patients with renal cell carcinoma undergoing nephrectomy with curative intent. Clin Genitourin Cancer 2016; 14: 271-6.

12. Yoshida T, Kinoshita H, Yoshida K, et al. Prognostic impact of perioperative lymphocyte-monocyte ratio in patients with bladder cancer undergoing radical cystectomy. Tumour Biol 2016; 37: 10067-74.

13. Osadnik T, Wasilewski J, Lekston A, et al. The plateletto-lymphocyte ratio as a predictor of all-cause mortality in patients with coronary artery disease undergoing elective percutaneous coronary intervention and stent implantation. J Saudi Hear Assoc 2015; 27: 144-51.

14. Kishimoto N, Takao T, Kuribayashi S, et al. The neutrophil-to-lymphocyte ratio as a predictor of intravesical recurrence in patients with upper urinary tract urothelial carcinoma treated with radical nephroureterectomy. Int J Clin Oncol 2017; 22: 153-8.

15. Yin Y, Kuai S, Liu J, et al. Pretreatment neutrophil-to-lymphocyte ratio in peripheral blood was associated with pulmonary tuberculosis retreatment. Arch Med Sci 2017; 13: 404-11.

16. Ozcicek A, Ozcicek F, Yildiz G, et al. Neutrophil-to-lymphocyte ratio as a possible indicator of epicardial adi- pose tissue in patients undergoing hemodialysis. Arch Med Sci 2017; 13: 118-23.

17. Viers BR, Houston Thompson R, Boorjian SA, et al. Preoperative neutrophil-lymphocyte ratio predicts death among patients with localized clear cell renal carcinoma undergoing nephrectomy. Urol Oncol 2014; 32: 1277-84.

18. Chang Y, Fu Q, Xu L, et al. Prognostic value of preoperative lymphocyte to monocyte ratio in patients with nonmetastatic clear cell renal cell carcinoma. Tumour Biol 2016; 37: 4613-20.

19. Xia WK, Wu X, Yu TH, et al. Prognostic significance of lymphocyte-to-monocyte ratio and CRP in patients with nonmetastatic clear cell renal cell carcinoma: a retrospective multicenter analysis. Onco Targets Ther 2016; 9: 2759-67.

20. Hu H, Yao X, Xie X, et al. Prognostic value of preoperative $\mathrm{NLR}, \mathrm{dNLR}, \mathrm{PLR}$ and CRP in surgical renal cell carcinoma patients. World J Urol 2017; 35: 261-70.

21. Proctor MJ, McMillan DC, Morrison DS, et al. A derived neutrophil to lymphocyte ratio predicts survival in patients with cancer. Br J Cancer 2012; 107: 695-9.

22. Edge SB, Compton CC. The American Joint Committee on Cancer: the $7^{\text {th }}$ edition of the AJCC cancer staging manual and the future of TNM. Ann Surg Oncol 2010; 17: 1471-4.

23. Fuhrman SA, Lasky LC, Limas C. Prognostic significance of morphologic parameters in renal cell carcinoma. Am J Surg Pathol 1982; 6: 655-63.

24. Gu L, Ma X, Li H, et al. Prognostic value of preoperative inflammatory response biomarkers in patients with sarcomatoid renal cell carcinoma and the establishment of a nomogram. Sci Rep 2016; 6: 23846.

25. Altman DG, McShane LM, Sauerbrei W, Taube SE. Reporting Recommendations for Tumor Marker Prognostic Studies (REMARK): explanation and elaboration. PLoS Med 2012; 9: e1001216.

26. Royston P, Altman DG, Sauerbrei W. Dichotomizing continuous predictors in multiple regression: a bad idea. Stat Med 2006; 25: 127-41.

27. Elinav E, Nowarski R, Thaiss CA, et al. Inflammation-induced cancer: crosstalk between tumours, immune cells and microorganisms. Nat Rev Cancer 2013; 13: 759-71.

28. Balkwill F, Mantovani A. Inflammation and cancer: back to Virchow? Lancet 2001; 357: 539-45.

29. Ray-Coquard I, Cropet C, Van Glabbeke M, et al. Lymphopenia as a prognostic factor for overall survival in advanced carcinomas, sarcomas, and lymphomas. Cancer Res 2009; 69: 5383-91.

30. Riedl J, Pabinger I, Ay C. Platelets in cancer and thrombosis. Hamostaseologie 2014; 34: 54-62.

31. Richards DM, Hettinger J, Feuerer M. Monocytes and macrophages in cancer: development and functions. Cancer Microenviron 2013; 6: 179-91.

32. Coffelt SB, Wellenstein MD, de Visser KE. Neutrophils in cancer: neutral no more. Nat Rev Cancer 2016; 16: 431-46.

33. Kim R, Emi M, Tanabe K, et al. The role of Fas ligand and transforming growth factor beta in tumor progression: molecular mechanisms of immune privilege via Fas-mediated apoptosis and potential targets for cancer therapy. Cancer 2004; 100: 2281-91.

34. Mantur M, Kemona H, Kozlowski R, Kemona-Chetnik I. Effect of tumor stage and nephrectomy on CD62P expression and $\mathrm{SP}$-selectin concentration in renal cancer. Neoplasma 2003; 50: 262-5.

35. Osadnik T, Strzelczyk JK, Lekston A, et al. The association of functional polymorphisms in genes encoding 
growth factors for endothelial cells and smooth muscle cells with the severity of coronary artery disease. BMC Cardiovasc Disord 2016; 16: 218

36. Osadnik T, Strzelczyk JK, Reguła R, et al. The Relationships between polymorphisms in genes encoding the growth factors TGF-beta1, PDGFB, EGF, bFGF and VEGF-A and the restenosis process in patients with stable coronary artery disease treated with bare metal stent. PLoS One 2016; 11: e0150500.

37. Li WL, Hua LG, Qu P, et al. NLRP3 inflammasome: a novel link between lipoproteins and atherosclerosis. Arch Med Sci 2016; 12: 950-8.

38. Komohara Y, Hasita H, Ohnishi K, et al. Macrophage infiltration and its prognostic relevance in clear cell renal cell carcinoma. Cancer Sci 2011; 102: 1424-31.

39. Lucca I, de Martino M, Hofbauer SL, et al. Comparison of the prognostic value of pretreatment measurements of systemic inflammatory response in patients undergoing curative resection of clear cell renal cell carcinoma. World J Urol 2015; 33: 2045-52.

40. Chen Z, Shao Y, Yao H, et al. Preoperative albumin to globulin ratio predicts survival in clear cell renal cell carcinoma patients. Oncotarget 2017; 8: 48291-302.

41. Pichler M, Hutterer GC, Stoeckigt C, et al. Validation of the pre-treatment neutrophil-lymphocyte ratio as a prognostic factor in a large European cohort of renal cell carcinoma patients. Br J Cancer 2013; 108: 901-7.

42. Dalpiaz O, Luef T, Seles M, et al. Critical evaluation of the potential prognostic value of the pretreatment-derived neutrophil-lymphocyte ratio under consideration of C-reactive protein levels in clear cell renal cell carcinoma. Br J Cancer 2017; 116: 85-90.

43. Hutterer GC, Stoeckigt C, Stojakovic T, et al. Low preoperative lymphocyte-monocyte ratio (LMR) represents a potentially poor prognostic factor in nonmetastatic clear cell renal cell carcinoma. Urol Oncol 2014; 32: 1041-8. 